\title{
Políticas, procesos y trayectorias de privatización educativa en Latinoamérica1
}

\section{Resumen}

\author{
Mauro Carlos Moschetti \\ ID ORCID: 0000-0002-0162-6860 \\ Clara Fontdevila ${ }^{2}$ \\ ID ORCID: 0000-0003-0589-558X \\ Antoni Verger $^{2}$ \\ ID ORCID: 0000-0003-3255-7703
}

Las políticas pro-privatización son un componente cada vez más importante en los procesos de reforma educativa en muchos puntos del planeta. América Latina es la región en la que la privatización educativa ha avanzado de forma más acusada en las últimas décadas. Sin embargo, la literatura académica sobre el tema es todavía escasa y son pocos los trabajos que adoptan una perspectiva regional. El objetivo de este artículo es desensamblar la naturaleza, condicionantes y variantes de la economía política de la privatización educativa en Latinoamérica, y establecer una tipología de trayectorias a través de las cuales avanza la privatización de la educación en dicha región. A partir de la triangulación de tres métodos de recolección de datos (la revisión y sistematización de 169 estudios sobre los procesos de privatización educativa en Latinoamérica, el análisis de fuentes estadísticas, y la realización de entrevistas con informantes clave) hemos identificado las siguientes trayectorias: 1) la privatización como parte de la reforma estructural del Estado; 2) la privatización como reforma incremental; 3) la privatización 'por defecto' y la emergencia de escuelas privadas de bajo coste; 4) las alianzas públicoprivadas históricas; 5) la privatización por vía del desastre; y 6) la privatización latente. Ahora bien, cabe tener en cuenta que en algunos países se produce una sedimentación de diferentes políticas y procesos pro-privatización donde convergen mecanismos característicos de más de una trayectoria.

\section{Pablaras clave}

América Latina - Privatización - Revisión sistemática de literatura - Política educativa Economía política.

1- La investigación que ha hecho posible este trabajo se ha desarrollado gracias al apoyo de Education International en el marco de la campaña Unite for Quality Education.

2- Universitat Autònoma de Barcelona, Barcelona, España.

Contactos: mauro.moschetti@uab.cat; clara.fontdevila@uab.cat; antoni.verger@uab.cat.

(c) (\$) DOl: http://dx.doi.org/10.1590/S1678-463420194187870

This content is licensed under a Creative Commons attribution-type BY-NC. 


\section{Policies, processes, and paths of educational privatization in Latin America}

\section{Abstract}

Pro-privatization policies are an increasingly important component in educational reform processes in many parts of the world. Latin America is the region in which educational privatization has advanced more sharply in recent decades. However, the academic literature on the subject is still scarce and there are few works that adopt a regional perspective. The objective of this article is to disassemble the nature, conditions and variants of the political economy of educational privatization in Latin America, and establish a typology of 'paths' through which educational privatization has advanced in the continent. Drawing on the triangulation of three methods of data collection (review and systematization of 169 studies on the processes of educational privatization in Latin America, analysis of statistical sources, and interviews with key informants), we have identified the following paths: 1) privatization as part of the structural reform of the State; 2) privatization as an incremental reform; 3) 'default' privatization and the emergence of low-cost private schools; 4) historical public-private partnerships; 5) privatization via disaster; and 6) latent privatization. In some countries there is a sedimentation of different pro-privatization policies and processes where elements of different paths tend to converge.

\section{Keywords}

Latin America - Privatization - Systematic literature review - Educational policy Political economy.

\section{Introducción}

Las políticas proprivatización están muy presentes en los procesos de reforma educativa de un número creciente de países y regiones (VERGER; FONTDEVILA; ZANCAJO, 2016). América Latina es la región en la que la privatización educativa, medida por la cantidad de alumnos en instituciones privadas, ha crecido de forma más acusada en las últimas décadas. El caso latinoamericano destaca no sólo por encabezar el ranking de regiones con mayor participación educativa privada, sino por ser además la región en la que el crecimiento ha sido más sostenido a lo largo de las últimas décadas (UNESCO-UIS, 2016) (véase Gráficos 1 e 2).

Entre 1990 y 2014, la mayoría de los países de la región ha transitado de niveles de privatización bajos a medios, o de niveles medios a altos en educación primaria. En el nivel secundario, si bien las variaciones interanuales son más heterogéneas, la participación educativa privada es muy elevada en virtualmente todos los países de la región -sobre 
Gráfico 1- Porcentaje de alumnos matriculados en instituciones privadas de nivel primario por regiones 1990-2014

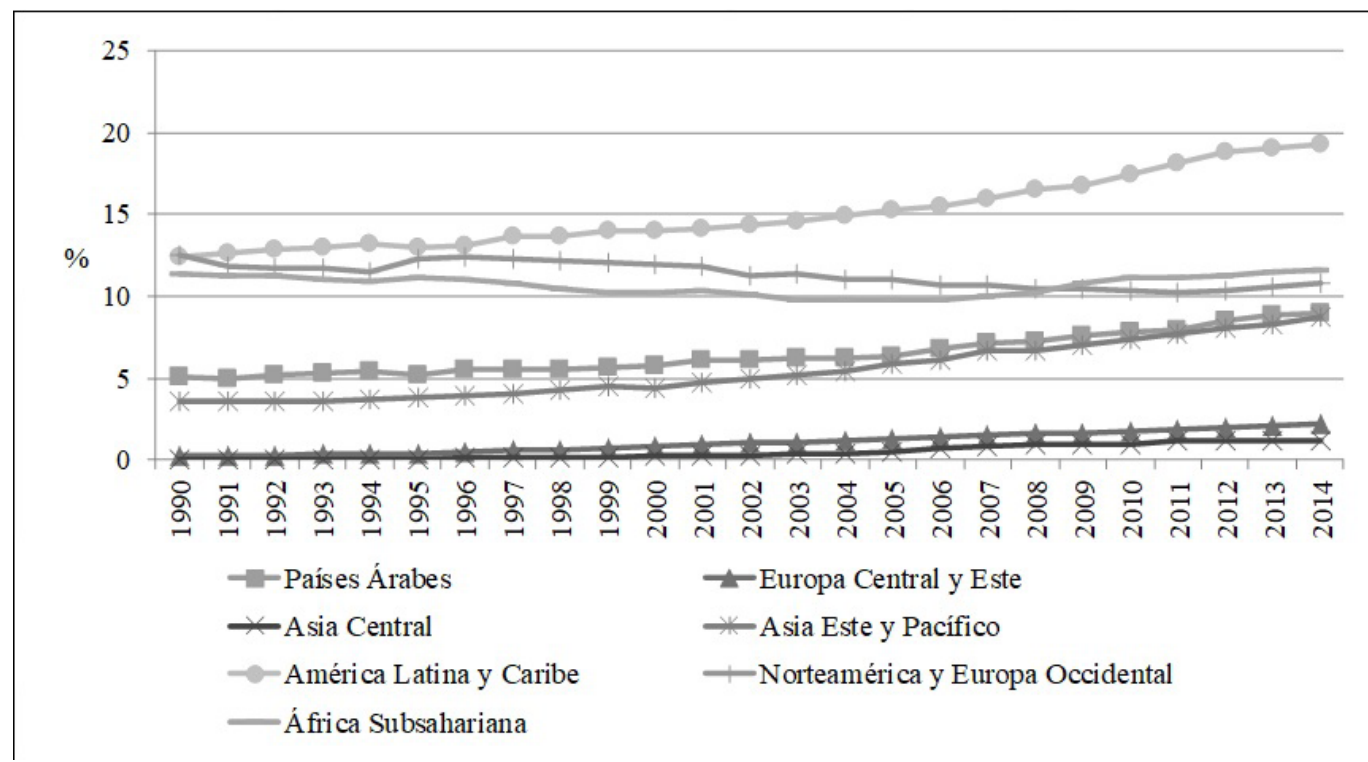

Fuente: Adaptado de la información del Instituto de Estadísticas de UNESCO (2016).

Nota: UNESCO considera instituciones privadas a aquellas que no son operadas por una agencia o autoridad pública sino que están controladas y gestionadas, ya sea con 0 sin fines de lucro, por alguna organización no gubernamental (por ejemplo, instituciones religiosas, sindicatos, empresas, fundaciones, 0 agencias extranjeras), 0 a aquellas cuya junta de gobierno está compuesta principalmente por miembros que no han sido seleccionados por un organismo público.

Gráfico 2- Porcentaje de alumnos matriculados en instituciones privadas de nivel secundario por regiones 1998-2014

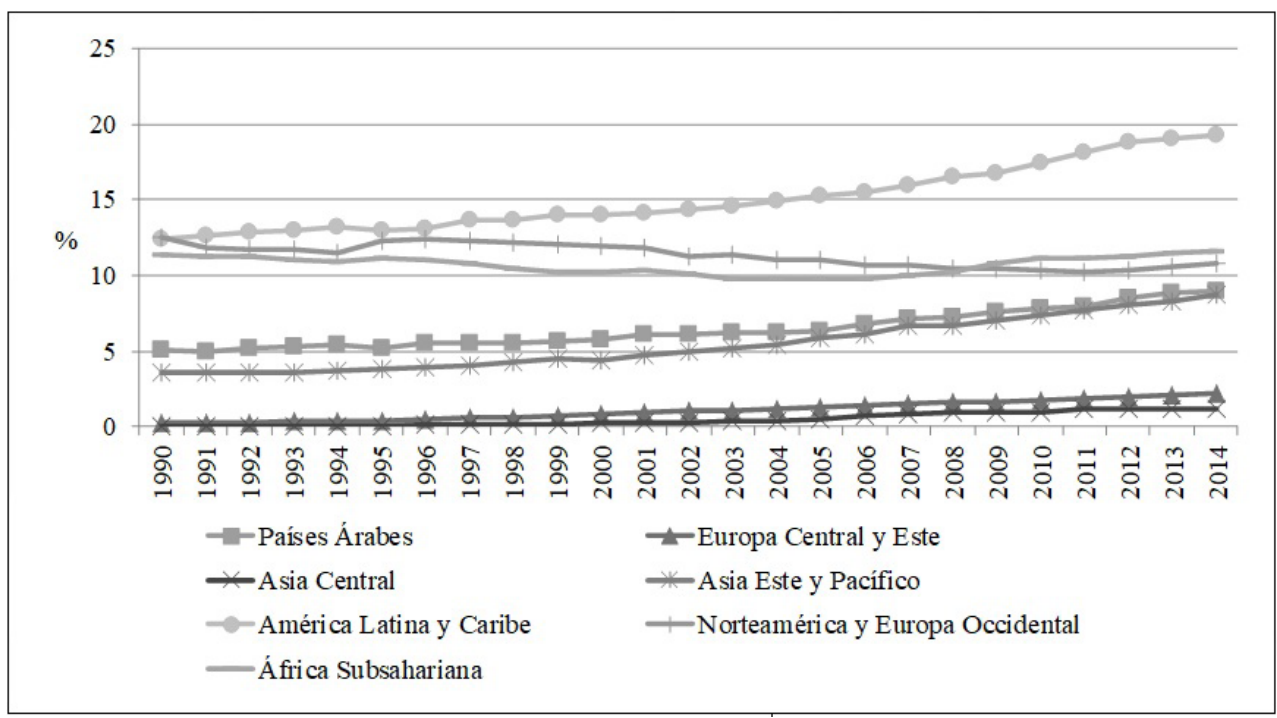

Fuente: Adaptado de la información del Instituto de Estadísticas de UNESCO (2016). 
todo si se compara con Europa, América del Norte y Asia, donde las tasas de privatización no suelen superar el 15\% de media (véase Tablas 1 e 2).

Tabla 1- Porcentaje de alumnos matriculados en instituciones privadas de nivel primario. Países seleccionados 1990-2000-2014

\begin{tabular}{|c|c|c|c|c|c|c|}
\hline País & $\%$ Privado 1990 * & $\%$ Privado 2000 ** & $\%$ Privado 2014 *** & \% Variación & 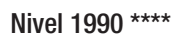 & Nivel 2014 \\
\hline Perú & 12.6 & 13.0 & 27.2 & 116.2 & B & M \\
\hline Brasil & 8.5 & 8.3 & 16.2 & 91.1 & B & M \\
\hline Costa Rica & 4.7 & 6.9 & 8.8 & 88.0 & B & B \\
\hline Jamaica & 4.8 & 5.2 & 8.5 & 78.5 & B & B \\
\hline Panamá & 7.8 & 9.9 & 13.8 & 76.1 & B & B \\
\hline Honduras & 5.8 & 6.1 & 9.8 & 71.1 & B & B \\
\hline Ecuador & 15.9 & 21.8 & 24.3 & 52.6 & M & M \\
\hline Chile & 41.9 & 46.5 & 60.2 & 43.6 & $A$ & $A$ \\
\hline México & 6.0 & 7.4 & 8.5 & 42.8 & B & B \\
\hline Argentina & 18.9 & 20.6 & 25.3 & 34.2 & $M$ & M \\
\hline Paraguay & 15.0 & 15.0 & 18.9 & 26.4 & $B$ & M \\
\hline Venezuela & 15.0 & 14.4 & 18.7 & 24.4 & M & M \\
\hline Nicaragua & 12.6 & 16.0 & 15.6 & 23.2 & B & $M$ \\
\hline Colombia & 15.2 & 18.8 & 18.7 & 22.5 & $M$ & $M$ \\
\hline Haití & 67.0 & 76.7 & 77.2 & 15.3 & I & 1 \\
\hline $\begin{array}{l}\text { República } \\
\text { Dominicana }\end{array}$ & 21.2 & 15.8 & 23.6 & 10.9 & M & M \\
\hline Uruguay & 16.2 & 14.0 & 16.1 & -0.3 & $M$ & $M$ \\
\hline El Salvador & 11.0 & 11.2 & 10.8 & -1.7 & B & B \\
\hline Guatemala & 16.2 & 12.8 & 11.1 & -31.2 & M & B \\
\hline Bolivia & 20.7 & 20.7 & 9.2 & -55.5 & $M$ & B \\
\hline
\end{tabular}

Fuente: Adaptado de la información de Instituto de Estadísticas de UNESCO (2016) y de World Bank Data (2016).

*Argentina (1988); Bolivia (2000); Brasil (1999); Chile (1997); República Dominicana (1991); Ecuador (1993); El Salvador (1998); Guatemala (1991); Haití (1992); Venezuela (1999).

** Honduras (1999); Chile (2002); Paraguay (2001); Haití (1998); República Dominicana (1999).

***Argentina, Bolivia, Brasil, El Salvador, México, Panamá (2013); Chile (2012); Paraguay (2011); Uruguay (2010).

*** Niveles: Bajo (inferior al 15\%); Medio (15-30\%); Alto (30-65\%); Integral (superior al 65\%).

Sin embargo, y a pesar del avance de la privatización educativa en América Latina, la literatura sobre el fenómeno es todavía limitada en la mayoría de países de la región, y los trabajos que adoptan una perspectiva regional comprehensiva son escasos (véanse excepciones en Bellei y Orellana, 2014 y Clade, 2014). Si bien el fenómeno de la privatización educativa constituye el objeto de estudio de un número creciente de investigaciones, en el caso de América Latina éstas se suelen centrar en casos nacionales 
específicos y raramente han abordado la cuestión desde una perspectiva comparada o en relación a tendencias más generales. La perspectiva regional a la investigación de la reforma educativa resulta de hecho particularmente difícil de articular dada la gran diversidad interna de la región latinoamericana.

Tabla 2- Porcentaje de alumnos matriculados en instituciones privadas de nivel secundario. Países seleccionados 1999-2007-2014

\begin{tabular}{|c|c|c|c|c|c|c|}
\hline País & \% Privado 1999 * & $\%$ Privado 2007 ** & 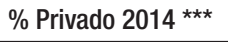 & \% Variación & 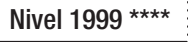 & Nivel 2014 \\
\hline Perú & 16.0 & 22.7 & 30.1 & 88.1 & M & $A$ \\
\hline Chile & 45.9 & 54.5 & 60.4 & 31.5 & A & A \\
\hline Ecuador & 24.3 & 32.0 & 29.8 & 22.9 & M & M \\
\hline Jamaica & 2.4 & 4.5 & 2.8 & 16.8 & B & B \\
\hline Brasil & 11.0 & 11.4 & 12.9 & 16.6 & B & B \\
\hline Uruguay & 12.7 & 12.5 & 14.5 & 14.5 & B & B \\
\hline Venezuela & 30.0 & 26.2 & 32.8 & 9.4 & M & A \\
\hline Panamá & 16.3 & 15.8 & 16.5 & 0.8 & M & M \\
\hline Argentina & 26.3 & 28.2 & 26.2 & -0.1 & M & $M$ \\
\hline Honduras & 27.4 & 26.3 & 26.0 & -5.0 & M & M \\
\hline México & 15.1 & 15.2 & 13.1 & -13.5 & M & B \\
\hline Guatemala & 73.5 & 74.0 & 62.3 & -15.2 & । & A \\
\hline $\begin{array}{c}\text { República } \\
\text { Dominicana }\end{array}$ & 23.1 & 22.1 & 19.5 & -15.9 & M & M \\
\hline Paraguay & 29.4 & 21.5 & 21.8 & -25.9 & M & M \\
\hline Costa Rica & 12.4 & 10.0 & 9.1 & -26.2 & B & B \\
\hline Nicaragua & 32.1 & 23.7 & 21.8 & -31.9 & A & M \\
\hline El Salvador & 24.9 & 18.5 & 16.9 & -32.2 & M & $M$ \\
\hline Colombia & 32.4 & 24.1 & 20.3 & -37.3 & A & $M$ \\
\hline Bolivia & 29.2 & 13.5 & 12.9 & -55.7 & $M$ & B \\
\hline Haití & $\mathrm{s} / \mathrm{d}$ & $\mathrm{s} / \mathrm{d}$ & $\mathrm{s} / \mathrm{d}$ & $\mathrm{s} / \mathrm{d}$ & $\mathrm{s} / \mathrm{d}$ & $\mathrm{s} / \mathrm{d}$ \\
\hline
\end{tabular}

Fuente: Adaptado de la información de Instituto de Estadísticas de UNESCO (2016) y de World Bank Data (2016).

* Bolivia (2000); Brasil, República Dominicana, Guatemala (2002); Chile, El Salvador (1998); Honduras (2006); Jamaica (2001); Uruguay (1998).

** Jamaica, Honduras, República Dominicana, Paraguay (2008).

***Argentina, Bolivia, Brasil, El Salvador, México, Panamá (2013); Chile, Paraguay (2012); Nicaragua, Uruguay (2010).

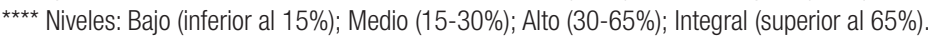

A raíz del carácter general del concepto de privatización educativa y de la heterogeneidad de políticas y procesos en las que se manifiesta, este estudio adopta una perspectiva de análisis centrada en la economía política de la reforma educativa. Dicha perspectiva requiere analizar de forma pormenorizada los condicionantes sociopolíticos, institucionales, demográficos e históricos de las reformas educativas emprendidas en distintos sistemas educativos nacionales y subnacionales -a la vez que analizar similitudes, diferencias y conexiones entre estas reformas. Los objetivos de este estudio son, por tanto, desensamblar la naturaleza, condicionantes y 
variantes de la privatización educativa en los diferentes territorios que conforman la región latinoamericana, y establecer una tipología de trayectorias de privatización de la educación a nivel regional, capaz de capturar no sólo los procesos formales de la privatización educativa, sino también su carácter construido y contextualizado.

El trabajo se organiza como sigue. En el primer apartado, presentamos las herramientas conceptuales y la metodología empleada en el estudio, tanto para la recolección de datos como para su análisis. En el segundo apartado, sistematizamos y exponemos las seis trayectorias hacia la privatización educativa que hemos identificado en América Latina. En el último apartado, discutimos los resultados del estudio y presentamos las principales conclusiones.

\section{Conceptos básicos y metodología}

\section{Delimitación del objeto de estudio}

Originalmente, los impulsores de las políticas proprivatización apuntaban a la necesidad de reconvertir los sistemas escolares estatales en sistemas de oferta privada sujetos a incentivos y a presiones competitivas (FRIEDMAN, 1955). Sin embargo, los procesos de privatización y desestatalización de los sistemas educativos (JESSOP, 2002) no han respondido en la práctica a una trayectoria única y se han caracterizado más bien por presentarse de manera "[...] compleja, multifacética e interrelacionada" (BALL, 2009, p. 83). Así, la privatización se ha manifestado típicamente en la constitución de sistemas educativos híbridos en los que el sector público y el privado interaccionan y se distribuyen responsabilidades de forma compleja y a menudo contradictoria (BELLEI; ORELLANA, 2014; MAROY, 2004).

En un trabajo ampliamente difundido, Ball y Youdell (2008) pretenden capturar esta heterogeneidad distinguiendo entre dos grandes tipos de privatización educativa. Distinguen así entre la privatización de tipo exógena, que se da cuando los Estados abren el sector público a la participación privada (incluyendo distintos esquemas de alianzas público-privada), y la privatización endógena, consistente en la importación al sector público de ideas y prácticas propias del sector privado, tales como las políticas de liderazgo empresarial, los incentivos financieros sujetos al desempeño, y, en general, diversas iniciativas ancladas en los lineamientos de la nueva gestión pública (COUPLAND; CURRIE; BOYETT, 2008). Con todo, es necesario tener en cuenta que, si bien esta conceptualización logra matizar la cada vez menos operativa distinción excluyente entre lo público y lo privado (BURCH, 2009), en la práctica, la privatización exógena y la endógena suelen presentarse de forma imbricada e híbrida (VERGER; FONTDEVILA; ZANCAJO, 2016).

En rigor, la complejidad descrita obliga a repensar la dicotomía público-privado y las formas de privatización educativa de modo gradual y contextualizado. A efectos de viabilidad y claridad conceptual, este estudio se centra fundamentalmente en el análisis de los procesos de privatización exógena ${ }^{3}$-independientemente de su posible conexión con otros procesos de naturaleza endógena.

3- Por ejemplo, México constituye en este sentido un caso excepcional en la región por cuanto las reformas educativas a lo largo de las últimas décadas han ido por la vía de la privatización endógena sin que se registraran variaciones de relevancia en términos de matriculación pública 0 privada (GERTLER; PATRINOS; RUBIO-CODINA, 2012; SANTIZO RODALL; MARTIN, 2009). 


\section{Aspectos metodológicos}

En vista de la diversidad interna que supone el concepto de privatización educativa, y a fin de obtener un mapeo de la situación de la privatización educativa en América Latina, este trabajo triangula tres métodos de recolección de datos complementarios. En primer lugar, la investigación se basa en una revisión y sistematización configurativa de la literatura sobre los procesos de privatización educativa en Latinoamérica (GOUGH; THOMAS; OLIVER, 2012). Concretamente, hemos revisado la producción científica sobre la temática contenida en libros y artículos académicos (a partir de las bases de datos SCOPUS y Google Scholar) y en literatura gris. La literatura revisada ha quedado circunscrita a los trabajos publicados entre 1990 y $2016^{4}$ y que tienen como foco principal el estudio de la privatización en los niveles de educación primaria y secundaria. El número de trabajos de investigación seleccionados para esta revisión ha sido de 169. A nivel de país investigado, estos trabajos se distribuyen de forma muy desigual (véase Tabela 3).

Tabla 3- Distribución de los estudios revisados por país/área geográfica

\begin{tabular}{|c|c|}
\hline País & Estudios revisados \\
\hline Argentina & 18 \\
\hline Bolivia & 9 \\
\hline Brasil & 18 \\
\hline Chile & 17 \\
\hline Colombia & 12 \\
\hline Costa Rica & 1 \\
\hline Cuba & 2 \\
\hline República Dominicana & 5 \\
\hline Ecuador & 4 \\
\hline El Salvador & 6 \\
\hline Guatemala & 2 \\
\hline Haití & 11 \\
\hline Honduras & 2 \\
\hline Jamaica & 3 \\
\hline México & 7 \\
\hline Nicaragua & 3 \\
\hline Paraguay & 1 \\
\hline Perú & 2 \\
\hline Uruguay & 9 \\
\hline Venezuela & 1 \\
\hline Comparativo & 38 \\
\hline Total & 169 \\
\hline
\end{tabular}

Fuente: Elaboración propia

4- El creciente interés por la temática en la región ha determinado que con posterioridad al año de cierre de esta revisión se hayan publicado algunos trabajos que dan cuenta de los procesos de privatización, en particular en Brasil y Argentina. Para el primero, destacan los trabajos de Siqueira y Nogueira (2017) y Perosa y Dantas (2017) que analizan el creciente fenómeno de proliferación de escuelas privadas de bajo costo dirigidas a sectores populares. Las aportaciones de estos trabajos apoyan la idea desarrollada en este artículo respecto de la coexistencia y sedimentación de políticas y procesos de privatización en los países de la región. También destaca el trabajo comparativo de Almeida y otros autores (2017) sobre los casos de Brasil y Argentina que contribuye a explicar la incidencia de los procesos de privatización educativa en materia de segmentación social. 
En segundo lugar, estos datos han sido complementados con información estadística sobre tasas de escolarización y porcentajes de participación del sector privado en la matriculación de alumnos, entre otros. Las fuentes de datos consideradas provienen primordialmente del Instituto de Estadística de la UNESCO y de las estadísticas educativas del Banco Mundial. Finalmente, hemos realizado entrevistas con informantes clave y expertos en contextos educativos específicos $(n=16)$ a efectos de triangular la información estadística y los datos obtenidos de la literatura académica, priorizando aquellos países sobre los cuales se dispone de menos literatura actualizada.

\section{Resultados: Trayectorias regionales hacia la privatización educativa}

A la hora de realizar la síntesis de los datos disponibles (literatura, estadísticas y entrevistas), nos hemos basado en la categorización de trayectorias de privatización educativa sistematizadas por Verger, Fondevila y Zancajo (2016) a escala global. Según estos autores, la privatización educativa se puede manifestar al menos en seis trayectorias: 1) como parte de la reforma estructural del Estado; 2) como reforma incremental; 3) la vía nórdica a la privatización educativa; 4) alianzas público-privadas históricas; 5) por defecto; y 6) por la vía del desastre. Estas trayectorias generales han sido adaptadas y redefinidas para el contexto de América Latina. Concretamente, la llamada vía nórdica, que se da eminentemente en países con estados del bienestar muy desarrollados, no aplica al contexto latinoamericano -en cambio, en América Latina identificamos una trayectoria poco estudiada a nivel global, a la que llamamos privatización latente.

La Tabla 1 recoge de modo esquemático los principales componentes de las trayectorias hacia la privatización educativa identificadas en América Latina. En los apartados siguientes, presentamos más detalladamente las principales características de cada una de estas trayectorias en base a los casos nacionales más representativos. Para cada una de las trayectorias, detallamos su origen, factores desencadenantes, principales resultados de políticas, y casos más emblemáticos.

Es importante apuntar que la tipología de trayectorias hacia la privatización propuesta en este artículo se plantea esencialmente como un ejercicio de categorización, sin pretensiones de exhaustividad. En este sentido, las trayectorias descritas deben entenderse en términos de tipos ideales o casos paradigmáticos, orientados a capturar y condensar los rasgos distintivos de diferentes procesos de privatización. Las diferencias entre trayectorias enfatizadas en el plano analítico no implican pues la ausencia de elementos transversales y comunes a todas ellas. Así mismo, las trayectorias descritas raramente se presentan de forma pura. Así, la privatización educativa avanza en la mayoría de los casos mediante la convergencia de elementos, mecanismos y actores característicos de varias de las trayectorias identificadas. 
Tabla 1- Resumen de resultados

\begin{tabular}{|c|c|c|c|c|}
\hline 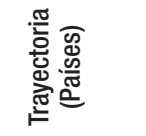 & Factores de privatización educativa & 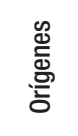 & Actores & Resultados \\
\hline 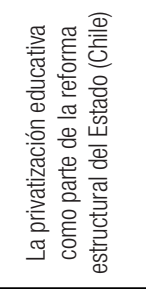 & $\begin{array}{l}\text { Orientación neoliberal de la dictadura militar y tecnocracia } \\
\text { asociada. } \\
\text { Autoritarismo y eliminación de oportunidades de veto. } \\
\text { Mecanismos implicados en la continuidad de las reformas: } \\
\text { Dificultades institucionales. } \\
\text { Presiones de familias y proveedores privados. } \\
\text { Evolución ideológica de las fuerzas de centro izquierda. } \\
\text { Negociación política con fuerzas conservadoras. } \\
\text { Condicionalidad préstamos del Banco Mundial. }\end{array}$ & 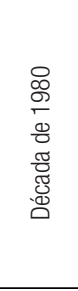 & $\begin{array}{c}\text { Junta Militar; gobiernos } \\
\text { tecnocráticos (dictadura } \\
\text { militar) } \\
\text { Gobiernos de centro } \\
\text { izquierda } \\
\text { Familias } \\
\text { Proveedores privados }\end{array}$ & $\begin{array}{l}\text { Sistema de financiamiento } \\
\text { per capita y mecanismos de } \\
\text { competición entre escuelas } \\
\text { Alto nivel de elección } \\
\text { escolar } \\
\text { Liberalización del sector } \\
\text { privado } \\
\text { Reformas de } \\
\text { endoprivatización } \\
\end{array}$ \\
\hline 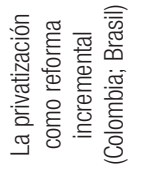 & $\begin{array}{l}\text { Adopción de la agenda de la nueva gestión pública. } \\
\text { Resistencia a las propuestas promercado más drásticas. } \\
\text { Modelo charter como second best (caso de Colombia). } \\
\text { Niveles altos de descentralización y desigualdades territoriales. } \\
\text { Concepción de la educación como servicio. }\end{array}$ & 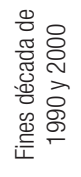 & $\begin{array}{l}\text { Gobiernos locales y } \\
\quad \text { estatales } \\
\text { Proveedores privados } \\
\text { Sindicatos docentes }\end{array}$ & $\begin{array}{c}\text { Alianzas público-privado } \\
\text { Elección escolar restringida, } \\
\text { pero pilotaje de modelos de } \\
\text { libre elección }\end{array}$ \\
\hline 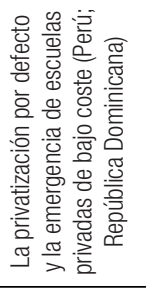 & $\begin{array}{c}\text { Incremento de la demanda educativa y ausencia o insuficiencia } \\
\text { de oferta estatal gratuita. } \\
\text { Masificación y superpoblación del sistema estatal en ámbitos } \\
\text { urbanos. } \\
\text { Rol ambiguo por parte del Estado en cuanto a la regulación del } \\
\text { sector privado/Desregulación de la actividad privada/Promoción } \\
\text { de esquemas de PPPs } \\
\text { Demandas desatendidas en cuanto a minorías religiosas. } \\
\text { Efecto legitimador por incremento de la cobertura escolar. }\end{array}$ & 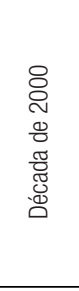 & $\begin{array}{l}\text { Pequeños y medianos } \\
\text { emprendedores locales } \\
\text { Organizaciones } \\
\text { internacionales } \\
\text { y corporaciones } \\
\text { transnacionales }\end{array}$ & $\begin{array}{l}\text { Proliferación de LFPSs } \\
\text { (individuales y pequeños y } \\
\text { grandes conglomerados) } \\
\text { Integración de LFPSs en } \\
\text { public-private partnerships } \\
\text { (PPPs). }\end{array}$ \\
\hline 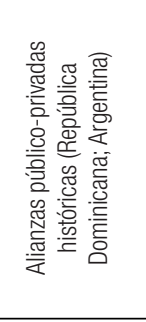 & $\begin{array}{l}\text { Relevancia histórica de instituciones religiosas en la educación. } \\
\text { Incremento de la demanda educativa y presiones para expandir } \\
\text { el acceso en contextos de crisis fiscal. } \\
\text { Marco de legitimación basado en la doctrina de la libertad de } \\
\text { enseñanza. } \\
\text { Amplio margen de autonomía institucional para los proveedores } \\
\text { privados. } \\
\text { Posterior extensión del esquema de PPP con proveedores no } \\
\text { religiosos. } \\
\text { Difícil reversibilidad económico-financiera. }\end{array}$ & 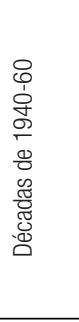 & $\begin{array}{l}\text { Instituciones religiosas } \\
\text { Asociaciones de } \\
\text { proveedores privados no } \\
\text { religiosos }\end{array}$ & $\begin{array}{c}\text { Subvenciones públicas } \\
\text { a escuelas privadas en } \\
\text { esquemas de PPP de plazo } \\
\text { indefinido } \\
\text { Desregulación y autonomía } \\
\text { pedagógica y administrativa } \\
\text { para escuelas privadas }\end{array}$ \\
\hline 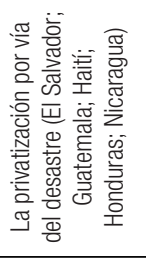 & $\begin{array}{c}\text { Efecto disparador de desastres naturales o conflictos armados; } \\
\text { crisis como campo de pruebas para la agenda de privatización. } \\
\text { Ausencia o atenuación del debate democrático a expensas del } \\
\text { estado de excepcionalidad. } \\
\text { Discursos de emergencia disipan los reparos y legitiman } \\
\text { reformas controversiales. } \\
\text { Vulnerabilidad estatal y fuerte presencia de agentes externos } \\
\text { (en particular, organismos multilaterales de crédito). }\end{array}$ & 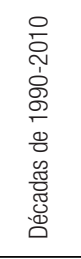 & $\begin{array}{l}\text { Agentes externos } \\
\text { (organismos multilaterales } \\
\text { de crédito, principalmente } \\
\text { el Banco Mundial y el } \\
\text { Banco Interamericano de } \\
\text { Desarrollo) }\end{array}$ & $\begin{array}{l}\text { Reformas de autonomía } \\
\text { escolar (SBM) } \\
\text { Expansión del rol de } \\
\text { las escuelas privadas } \\
\text { subvencionadas por vía de } \\
\text { vouchers }\end{array}$ \\
\hline 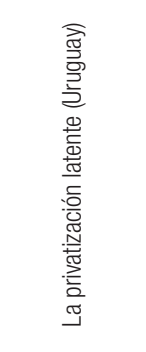 & $\begin{array}{c}\text { Desinversión estatal y pérdida de prestigio de la enseñanza } \\
\text { pública. } \\
\text { Tradición histórica de exoneración fiscal total para instituciones } \\
\text { religiosas a pesar de una fuerte tradición laica. } \\
\text { Establecimiento de exoneraciones tributarias parciales a las } \\
\text { donaciones privadas de empresas e instituciones privadas. } \\
\text { Impulso a la cooperación público-privado en materia de } \\
\text { infraestructura en un contexto de restricciones presupuestarias. } \\
\text { Soporte a fórmulas de subvención pública por parte de fuerzas } \\
\text { social demócratas (evolución ideológica; impacto tendencias } \\
\text { globales). }\end{array}$ & 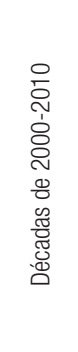 & $\begin{array}{c}\text { Asociaciones de } \\
\text { proveedores privados } \\
\text { (religiosos y no religiosos) } \\
\text { Think tanks, consultorías y } \\
\text { fundaciones } \\
\text { Partidos políticos de } \\
\text { distinto signo }\end{array}$ & $\begin{array}{l}\text { PPPs de infraestructura } \\
\text { Subvención indirecta a } \\
\text { centros de gestión privada } \\
\text { mediante exoneración } \\
\text { impositiva }\end{array}$ \\
\hline
\end{tabular}

Fuente: Elaboración propia. 


\section{La privatización educativa como parte de la reforma estructural del Estado}

En los casos de privatización educativa más drásticos, la privatización adopta un carácter estructural, y conlleva la redefinición profunda del papel del Estado en materia de financiación, provisión y regulación de la educación. A raíz de esta redefinición, el Estado pasa de proveer educación de forma directa a adoptar un rol centrado en la supervisión y distribución de incentivos.

El proceso de privatización emprendido por Chile es uno de los máximos exponentes de esta trayectoria hacia la privatización. Una profunda reforma de la educación primaria y secundaria implementada durante la dictadura militar convirtió a este país en un caso pionero de aplicación ortodoxa de la doctrina neoliberal en el campo educativo. Tal empresa se articuló fundamentalmente sobre un sistema de vouchers que con el tiempo adoptó un carácter cuasi-universal, llegando a financiar el 90\% de la matrícula primaria y secundaria en el país (PAREDES; UGARTE, 2009). Otros elementos clave de la reforma fueron la descentralización de la gestión de las escuelas públicas hacia las municipalidades (BELLEI, 2007) y la desregulación del trabajo docente.

Un primer elemento para comprender el calado de la reforma promercado en Chile es la fuerte influencia de las teorías económicas neoliberales y monetaristas, desarrolladas bajo el influjo de la Escuela de Chicago y especialmente del economista Milton Friedman. El compromiso ideológico de la dictadura de Augusto Pinochet (1973-1990) con la doctrina neoliberal explicaría la adopción temprana, el rápido avance y el marcado origen político de las reformas en las que dicha doctrina derivó (FOURCADE-GOURINCHAS; BABB, 2002).

Más allá de factores de carácter eminentemente ideológico, existen otros elementos que propiciaron también la aplicación de una agenda de reformas educativas tan ambiciosa. Por un lado, el crecimiento económico relativamente bajo experimentado por Chile durante los años 60 y 70, junto con los elevados niveles de inflación, contribuyeron a incrementar una cierta tensión social que, en última instancia, favorecieron la legitimación del carácter radical de las reformas emprendidas (FOURCADE-GOURINCHAS; BABB, 2002). Por otro lado, el contexto de dura represión política propio de la dictadura imposibilitó la articulación de una oposición efectiva a dichas reformas (DELANNOY, 2000; GAURI, 1998).

El retorno a la democracia en 1990 levantó muchas expectativas sobre una posible reversión de este conjunto de reformas promercado. Sin embargo, la dificultad estructural para revertir este tipo de reformas determinó que el sistema lograra sobrevivir al cambio de régimen (CARNOY, 2003). La coalición de centro izquierda surgida de las primeras elecciones democráticas mantuvo inalteradas las principales características del modelo de mercado durante las dos décadas en que se mantuvo en el poder. Si bien se intentó dar respuesta a las deficiencias de calidad y equidad del sistema, este objetivo se abordó en la práctica desde una perspectiva compensatoria, sin alterar la centralidad de los mecanismos de mercado (MIZALA, 2007).

La longevidad del régimen de mercado se explica por una compleja combinación de factores. En primer lugar, la promoción de la participación de actores privados en el sistema educativo tuvo como consecuencia la articulación de una serie de grupos de interés (proveedores privados, familias) con capacidad de ejercer presión a favor del 
mantenimiento del sistema de mercado (CARNOY, 2003; VALENZUELA; BELLEI; RIOS, 2014). En segundo lugar, las tensiones internas experimentadas dentro de los sucesivos gobiernos socialdemócratas impidieron el retorno a un sistema nacional de educación pública promovido por algunas facciones (BELLEI; VANNI, 2015; GAURI, 1998). En tercer lugar, destacan constreñimientos de carácter regulatorio, como la Ley Orgánica Constitucional de Enseñanza, aprobada en la etapa final de la dictadura y de difícil revocación en virtud de su categoría orgánica. Por otra parte, las condicionalidades de los préstamos del Banco Mundial contribuyeron también a la continuidad del esquema de mercado educativo (COX; ÁVALOS, 1999).

Finalmente, la aprobación de la política del copago en el año 1993, como resultado de las presiones de la oposición conservadora (MIZALA, 2007), representa un punto de inflexión en el aumento de la privatización educativa. Esta ley, que habilitaba el cobro de cuotas escolares adicionales a las escuelas privadas subvencionadas, supuso la consolidación y expansión de la provisión privada al convertir la educación en un sector económico potencialmente lucrativo (CARNOY, 2003).

Recientemente, la elevada complejidad de cualquier forma de reversión de la privatización en Chile se ha puesto de claro manifiesto a raíz de las medidas promovidas desde 2014 por el gobierno de centroizquierda orientadas a disminuir la centralidad de las dinámicas de mercado en educación frente a las demandas crecientes de la sociedad y los movimientos estudiantiles (CABALIN, 2012). Como señala Bellei (2016), estas propuestas se han enfrentado a fuertes resistencias, de naturaleza tanto pragmática (dependencia de la provisión privada) como ideológica (prevalencia de una concepción de la educación como bien privado).

\section{La privatización como reforma incremental}

En algunos países, la privatización educativa no es el resultado de cambios drásticos ni de reformas estructurales, sino que de la adopción de cambios más bien graduales, que se dan a menudo a nivel subnacional y, en cierta manera, de forma desconectada. No obstante, la acumulación de dichos cambios parciales acaba por alterar significativamente la constitución y la gobernanza del sistema de educación pública. En Latinoamérica, estos procesos pueden observarse más claramente en Colombia y Brasil, casos que comparten las siguientes características.

En primer lugar, se trata de países muy descentralizados y con muchas desigualdades territoriales, de manera que resulta complicado impulsar reformas educativas profundas a nivel nacional. Además, el proceso de descentralización de la administración pública no siempre ha resuelto de forma clara la distribución de responsabilidades educativas entre el gobierno nacional, estados y municipios. El gobierno federal o nacional tiene la capacidad de incentivar la provisión y el consumo de educación privada a través de medidas fiscales (véase Rezende Pinto, 2016, para el caso brasileño), pero no de imponer reformas concretas sobre la gobernanza del sistema escolar.

En segundo lugar, a nivel jurídico-legal, se trata de países que no sólo reconocen el rol del sector privado en educación, sino que también favorecen que el Estado 
subcontrate educación privada. En Colombia, la Ley General de Educación de 1994 permite compatibilizar la gratuidad de la educación con el cobro de derechos académicos a quienes puedan costearlos, así como con la obligación del Estado de contratar entidades educativas privadas en caso de identificar insuficiente oferta pública (TERMES et al., 2015). En Brasil, la constitución de 1988 garantiza la libertad de enseñanza y la iniciativa privada, en caso de que dicha iniciativa cumpla las normas generales de la educación nacional y sea autorizada por los poderes públicos (REIS DE FIGUEREID0, 2016). Además, la reforma educativa del primer gobierno de Cardoso en 1996, con el objetivo de modernizar el sistema público, otorgó mayores niveles de competencia y responsabilidad financiera a los municipios y permitió a escuelas privadas sin fines de lucro percibir fondos públicos (BELLEI; ORELLANA, 2014).

En tercer lugar, se trata de países en los que prevalecen altos niveles de desigualdades sociales, una infrafinanciación histórica del sector educativo público y una regulación restrictiva de la elección escolar. La combinación de estos factores favorece una cierta huida de las capas medias hacia el sector educativo privado (DA COSTA; KOSLINSKI; COSTA, 2013).

Finalmente, se trata de países en los que las reformas promercado son difíciles de implementar debido a circunstancias institucionales, pero también de carácter político, entre las que destaca la resistencia de los sindicatos docentes. En el caso de Brasil, los gobiernos difícilmente pueden prescindir, en términos políticos, del apoyo de organizaciones sindicales, incluidos las docentes - abiertamente contrarias a tales reformas (BROOKE, 2006). En Colombia los intentos de implementar un sistema de vouchers a principios de la década de 2000 fueron abandonados al advertir que la propuesta chocaría con la fuerte oposición de los partidos de izquierda y del influyente sindicato de personal docente del país, la Federación Colombiana de Educadores (TERMES et al., 2015).

A raíz de este tipo de resistencias, tanto explícitas como latentes, países como Colombia y Brasil son más proclives a la adopción de reformas promercado de carácter más moderado, como son las escuelas charter (TERMES et al., 2015), o medidas de privatización endógena, como son las políticas de incentivo docente y, en general, las medidas de financiación escolar en base a resultados (DA SILVA; ALVES, 2012). Este tipo de políticas se tienden a implementar en un marco territorial acotado y, del mismo modo que pueden expandirse a otros territorios (como ha sido el caso de las escuelas charter en Colombia), también pueden acabar desapareciendo, sin superar el estadio de prueba piloto.

\section{La privatización por defecto y la emergencia de escuelas privadas de bajo coste}

En un número importante de países del Sur global, el sector privado se ha expandido considerablemente en las últimas décadas sin que existieran políticas proprivatización. En estos casos, el incremento de la demanda educativa, la ausencia de sistemas educativos universales y de calidad, y la escasa capacidad de respuesta estatal, abren una ventana para el desarrollo de la actividad privada (TOOLEY; DIXON, 2006). La privatización se produce así por defecto, y se canaliza a menudo a través de las llamadas escuelas privadas de bajo coste (en inglés low-fee private schools, o LFPSs). Estas escuelas constituyen un tipo 
distintivo de escuelas privadas. Se trata a menudo de escuelas impulsadas y controladas por un individuo o un grupo de individuos, generalmente con fines lucrativos, y que ofrecen educación básica a familias de bajos ingresos a cambio de cuotas relativamente asequibles (PHILLIPSON, 2008; WALFORD, 2011).

Algunos investigadores argumentan que el avance de esta modalidad de escolarización estaría asociada a una supuesta superioridad de las LFPSs en materia de calidad en comparación con las escuelas estatales -lo cual sería un determinante en la elección escolar de muchas familias (TOOLEY; DIXON, 2006). No obstante, numerosos estudios señalan la mayor importancia de otros factores, como la falta de recursos, masificación o no disponibilidad de escuelas estatales gratuitas y, en ciertos casos, las preferencias lingüísticas o religiosas de algunos grupos sociales, que impulsarían a muchas familias a optar por LFPSs y descartar la escuela pública (HÄRMÄ, 2013; SARANGAPANI; WINCH, 2010; SRIVASTAVA, 2008). Desde una perspectiva de equidad, la alegada asequibilidad de las LFPSs resulta también una cuestión controvertida. En la mayoría de los casos, las cuotas no llegan a ser lo suficientemente bajas como para no excluir a los sectores más pobres (DAY ASHLEY et al., 2014).

En América Latina, países como Perú o la República Dominicana han experimentado en las últimas décadas una fuerte expansión del sector LFPS $^{5}$, como consecuencia de un conjunto de elementos contextuales comunes. En primer lugar, la expansión del sector LFPS se ha dado especialmente en zonas urbanas y urbano-periféricas con un fuerte crecimiento poblacional y de la demanda educativa. Así, en la ciudad de Lima la participación privada en la educación básica pasó del 29\% en 2004 al 50\% en 2014 a instancias de la veloz multiplicación de LFPSs (BALARIN, 2016). En República Dominicana, el proceso ha tenido como escenario principal a la ciudad de Santo Domingo, merced a un crecimiento demográfico sin precedentes en las últimas décadas del siglo XX (FLORES, 1997; GUZMÁN; CRUZ, 2009).

En segundo lugar, la falta de inversión estatal en educación resultó en un importante déficit de oferta pública, agravado por los cambios sociodemográficos y el consecuente incremento en la demanda concentrada territorialmente. En República Dominicana, por ejemplo, la inversión estatal en educación entre 1970 y 1995 rara vez superó el 2\% del PIB. El resultante déficit acumulado de plazas condujo a la superpoblación de las escuelas estatales, revertida sólo parcialmente entre 1993 y 2005 mediante el Plan Decenal de Educación de 1993 (GUZMÁN; CRUZ, 2009; UNESCO-UIS, 2016).

En tercer lugar, en esos contextos los Estados suelen adoptar posiciones ambiguas o de tolerancia respecto del crecimiento del sector LFPS y su regulación (SRIVASTAVA, 2008). De hecho, muchas LFPSs no suelen estar acreditadas. Generalmente, se valen de la contratación de docentes no cualificados, y del pago de salarios inferiores a los que perciben los docentes agremiados del sistema estatal. En República Dominicana, por ejemplo, Flores advierte que "[...] se ha requerido una elevada dosis de tolerancia para evitar el cierre masivo de establecimientos educativos que, aún en forma precaria, ofrecen

5- Recientes estudios indican que, aunque en un grado más incipiente que el evidenciado en Perú y República Dominicana, esta tendencia estaría comenzando a tomar cierto impulso en Brasil como resultado de la mejora en las condiciones económicas de un sector de las clases populares durante la década pasada (PEROSA; DANTAS, 2017; SIQUEIRA; NOGUEIRA, 2017). 
un servicio educativo que el Estado no puede cubrir" (1997, p. 10). En Perú, las LFPS operan también en un contexto muy desregulado, e incluso lo hacen bajo el control formal del Instituto de Defensa del Consumidor en lugar del Ministerio de Educación (BALARIN, 2016). Existe en todo caso un efecto legitimador de la oferta privada basado en su efecto positivo sobre la tasa de cobertura.

\section{Alianzas público-privadas históricas}

Algunos países cuentan con sistemas educativos duales público-privados como resultado de una larga tradición de interacción entre el Estado y corporaciones religiosas en el campo de la provisión educativa. Se trata de países cuyos Estados, en sus intentos por instaurar o expandir sus sistemas educativos, optaron por establecer alianzas estables con entidades religiosas que posteriormente se extenderian a otros tipos de proveedores privados. Dichas alianzas se produjeron con bastante anterioridad y de manera desconectada de la emergencia de las políticas y discursos neoliberales de los años ochenta. En América Latina, este tipo de alianzas público-privadas (o PPP por sus siglas en inglés, publicprivate partnerships) están muy arraigadas en República Dominicana y Argentina.

En República Dominicana, el sector privado religioso ha sido un aliado del Estado en la provisión educativa desde mediados del siglo XX (GUZMÁN; CRUZ, 2009). En el marco del aún vigente Concordato de 1954 firmado entre el Vaticano y la República Dominicana, se institucionalizó un régimen de financiamiento estatal para las escuelas católicas inspirado en el sistema holandés. Como consecuencia de dicho tratado y de la escasa inversión estatal en infraestructura escolar pública, el sistema educativo dominicano cuenta con numerosas escuelas públicas administradas por privados $\mathrm{y}$ escuelas semioficiales (FLORES, 1997). Las primeras son de propiedad estatal, pero están gestionadas en su mayoría por la Iglesia Católica. Las segundas son escuelas de titularidad privada, también generalmente católicas, a las que el Estado transfiere fondos públicos para el pago de hasta un 90\% de los salarios docentes. Recientemente, el Ministerio de Educación y el Episcopado dominicanos firmaron un controvertido convenio marco, actualmente en proceso de reglamentación e implementación (TEJADA, 2015), que tiende a profundizar esta alianza habilitando a toda escuela católica semioficial a pasarse al régimen público de subvención estatal total.

En el caso de Argentina el proceso de configuración de una amplia PPP en el campo educativo cuenta con características y tiempos algo diferentes. En este país el período comprendido entre la organización del Estado nacional y comienzos de la década de 1950 coincide con una sostenida contracción del sector privado frente a la fuerte expansión de la educación estatal (NAHON, 2002). Así, hacia 1945-50, el sector privado tan solo representaba el 7\% de la matrícula del nivel primario (MORDUCHOWICZ; IGLESIAS, 2011). Sin embargo, a partir del período 1950-1960, comienza un proceso de crecimiento de la matrícula en el sector privado que se sostiene hasta la actualidad, y que alimenta dinámicas de fragmentación de la oferta educativa con impacto negativo sobre la cohesión social (PEREYRA, 2008; MORDUCHOWICZ, 2001; NARODOWSKI; MOSCHETTI, 2015). 
El origen del proceso de privatización se halla en la institucionalización de un sistema de subvenciones estatales a la educación privada en el año 1947 (Ley 13.047) (VIOR; RODRÍGUEZ, 2012). El sistema de subvenciones se mantuvo inicialmente muy acotado, teniendo por objetivo original combatir la inestabilidad laboral de los docentes del sector privado en retracción -fundamentalmente católico- frente a las conquistas gremiales para el personal estatal (CUCUZZA, 1997). Sin embargo, entre las décadas de 1950 y 1990, la normativa fue progresivamente extendida asegurando una mayor participación del Estado en el subsistema privado. La ampliación del sistema de subvenciones coincidió a su vez con un proceso de desregulación y autonomización del sector privado (MORDUCHOWICZ, 2001; NARODOWSKI; ANDRADA, 2001), que avanzó en paralelo a un proceso de legitimación de la provisión privada en base al principio de libertad de enseñanza y la crítica al intervencionismo estatal (VIOR; RODRÍGUEZ, 2012). El sistema de subvenciones al sector privado habría adquirido así un carácter estructural hasta tal punto que, en la actualidad, cada gobierno provincial financia entre el $40 \%$ y el $100 \%$ del gasto en salarios docentes del 65\% de las escuelas privadas del país, tanto religiosas como no religiosas (MEZZADRA; RIVAS, 2010; MOSCHETTI, 2015).

Recientemente, la expansión del sector privado se ha visto beneficiada por el crecimiento económico del período de post crisis (2001-2002) (BOTTINELLI, 2013), y especialmente de la relativa redistribución de dicho crecimiento en beneficio de los sectores de clase media y media-baja emergentes (GAMALLO, 2011; JUDZIK; MOSCHETTI, 2016). En la actualidad el sector privado representa cerca del 30\% de la matrícula total para educación obligatoria, sobrepasando el 40\% en grandes centros urbanos (DINIECE, 2016).

En definitiva, la PPP que ha configurado el sistema educativo argentino durante las últimas siete décadas resulta del efecto sedimentario de las gestiones y opciones de gobiernos diferentes (durante períodos tanto democráticos como no democráticos), que han dado apoyo y consolidado por acción u omisión dicho sistema de provisión dual (GAMALLO, 2015; GOTTAU; MOSCHETTI, 2015; VIOR; RODRÍGUEZ, 2012). Tal alianza con el sector privado se erige, además, en un contexto de restricciones presupuestarias fiscales cada vez más frecuentes y ante la necesidad de extender la cobertura educativa (NARODOWSKI, 2008). Así, una suma de factores augura su difícil reversión: la percepción del esquema como costo-eficiente desde la perspectiva del Estado, la dependencia del sector privado en las jurisdicciones en que este cubre entre el 30 y el 50\% de la matrícula total, y el poder de lobby de ciertas asociaciones de centros privados, junto con la presencia de representantes del sector privado en los organismos de supervisión de las escuelas no estatales (DE LUCA, 2008; PUIGGRÓS, 2003).

\section{La privatización por la vía del desastre}

En algunos casos, la privatización educativa avanza en el marco de catástrofes o crisis profundas. Situaciones de emergencia, desastres naturales o conflictos armados a menudo constituyen un marco propicio para la adopción de reformas educativas drásticas que, en condiciones normales, avanzarían con mayor dificultad. En América Latina, los casos de El Salvador, Nicaragua, Guatemala, Honduras y Haití ejemplifican esta trayectoria. 
A pesar de su gran diversidad, estos casos comparten una serie de elementos que dan consistencia a la trayectoria. En primer lugar, los episodios de crisis humanitaria permiten una importante presencia e influencia de agentes externos que conciben los territorios afectados por la crisis como campos de pruebas de soluciones y políticas innovadoras. En segundo lugar, en contextos poscatástrofe, el debate democrático sobre opciones y alternativas políticas suele atenuarse como consecuencia de la emergencia que prima para restituir la situación de normalidad. Dicho sentimiento de urgencia facilita la adopción de reformas drásticas o que, en situaciones de estabilidad, generarían más debate y controversia. Finalmente, destaca la capacidad de amplificación que ofrecen estos contextos, es decir, la alta probabilidad de que las reformas implementadas tengan un impacto más allá del territorio propiamente afectado por el desastre, así como de perdurar en el tiempo (VERGER; FONTDEVILA; ZANCAJO, 2016).

Durante la década de 1990, El Salvador, Nicaragua, Guatemala y Honduras adoptaron reformas educativas similares introducidas bajo condiciones políticas y sociales muy adversas y a instancias de las recomendaciones y el financiamiento provistos por agencias internacionales. Estas reformas se centraron en la adopción de programas de descentralización educativa y autonomía en la gestión escolar (en inglés: school-based management, o SBM) -todas ellas implementadas durante un período en el que las reformas prodescentralización enfrentaban una fuerte oposición en la región (EDWARDS, 2015; GANIMIAN, 2016).

Con algunas diferencias, los distintos programas de SBM implementados en Centroamérica se caracterizaron por un marcado corte eficientista, expandiendo el acceso a la educación con importantes ahorros derivados del trabajo no remunerado de las asociaciones de padres, el uso de infraestructuras no siempre concebidas para fines escolares y, fundamentalmente, el pago de salarios docentes más bajos. Se trató pues de formas de privatización endógena, con importantes efectos de deslegitimación de la provisión estatal y de debilitamiento de los sindicatos docentes (CUÉLLAR-MARCHELLI, 2003).

Significativamente, los distintos programas de SBM implementados en la región fueron adoptados en un contexto de suspensión del debate democrático. En El Salvador, la guerra civil (1980-1992) sirvió de base para la implementación del programa EDUCO (Educación con Participación de la Comunidad) durante la etapa final del conflicto. Así, el primer préstamo del Banco Mundial utilizado para la implementación del programa fue acordado en 1991 -previamente a los Acuerdos de Paz de enero de 1992, imposibilitando así un debate abierto y democrático (GUZMÁN; MEZA; DE VARELA, 2004).

Una dinámica similar se observa para el caso de Nicaragua, que lanzó en 1993 el Programa de Escuelas Autónomas, tras dos décadas de conflicto y en un contexto de capacidad económica limitada. También en este caso la influencia externa tuvo un papel clave en el avance de la reforma. Dicho programa se benefició de la asistencia del Banco Mundial, el Banco Interamericano de Desarrollo (BID) y la agencia norteamericana de ayuda internacional (USAID), y fue implementada por una coalición de gobierno liberal con el apoyo explícito del gobierno estadounidense (CASTILLO; MARTÍNEZ, 2015).

En Guatemala, el PRONADE (Programa Nacional de Autogestión para el Desarrollo Educativo) tuvo también su origen en la fase final del conflicto armado que vivió el país 
durante 36 años, y recibió apoyo técnico y financiero del Banco de Desarrollo Alemán, del gobierno francés y del Banco Mundial. Sin embargo, cabe señalar que esta ayuda nunca fue tan significativa como la que recibieron otros países de la región para la implementación de reformas de SBM (GANIMIAN, 2016).

En el caso en Honduras, el PROHECO (Proyecto Hondureño de Educación Comunitaria) fue adoptado más tardíamente (1999) y estuvo íntegramente financiado por el Banco Mundial. Con los sindicatos docentes abocados en ese momento en la lucha por el cumplimiento de los incrementos salariales fijados por el estatuto docente y en un contexto de crisis desatado por el huracán Mitch, el Banco Mundial pudo promover abiertamente y sin apenas resistencia la autogestión escolar como forma de recomponer el sistema educativo (YITZACK PAVÓN, 2008).

Finalmente, en Haití, la devastación derivada del terremoto que asoló al país en 2010 fue clave no tanto en la expansión del sector privado -históricamente mayoritario y heterogéneo, y que ya representaba el 80\% de la matrícula en la década de 2000 (DEMOMBYNES; HOLLAND; GIANMARCO, 2010; VALLAS; PANKOVITS, 2010)-, sino en su consolidación e integración en esquemas de alianza público-privada. En efecto, el terremoto y la consecuente necesidad de reconstruir el sistema educativo habrian transformado en políticas deliberadas un fenómeno que, hasta la fecha, se había dado de facto. Concretamente, los programas de reconstrucción implementados en Haití se centraron en la remodelación e integración del sector privado mediante un sistema de subsidios, una parte importante de los cuales se canalizó a través del plan anunciado en marzo de 2010 por el BID, que devino el principal partner del gobierno en la reconstrucción del sistema educativo (MCNULTY, 2011; 0'KEEFE, 2010; VALLAS; PANKOVITS, 2010).

\section{La privatización latente}

Existe una última trayectoria hacia la privatización educativa que se da en países donde la educación privada ha conocido históricamente un desarrollo muy limitado. Durante las últimas décadas, en estos países se observan síntomas de un cierto impulso de lógicas promercado que denotan una relativa penetración de la agenda privatizadora ampliamente extendida en la región. Esta trayectoria, que denominamos privatización latente, tiene en Uruguay su caso más emblemático. En este país, el sector público conserva un rol central en la provisión educativa y, desde un punto de vista histórico, la educación privada ha tenido un peso relativamente pequeño y con un claro componente de élite, oscilando entre el 10\% y el 15\% de la matrícula educativa a lo largo de todo el siglo XX (BELLEI; ORELLANA, 2014; BETANCUR, 2008; MANCEBO, 2008; INEED, 2015). En gran medida, el escaso desarrollo del sector privado en la educación uruguaya obedece a la fuerte tradición laica del país, ligada a la temprana separación Iglesia-Estado (DA COSTA, 2009) -si bien en la misma Constitución de 1918 se estableció la exoneración tributaria para las instituciones religiosas, alentando indirectamente una cierta pervivencia de los establecimientos educativos vinculados a órdenes religiosas. 
Si bien el rol marginal del sector privado se explica históricamente por el peculiar acuerdo Iglesia-Estado, las causas del escaso crecimiento del sector privado durante las décadas de los 80 y los 90 y de la poca penetración de la agenda neoliberal en el campo educativo resultan más inciertas. La literatura revisada apunta a explicaciones de naturaleza variada, entre las cuales destacan: 1) las preferencias ideológicas personales de los impulsores y gestores de la reforma educativa, que optaron por una reforma liberal amortiguada, a pesar del signo conservador del gobierno de la época (BETANCUR, 2008); 2) el papel clave de la escuela pública en el proceso de construcción del Estado moderno uruguayo y su asociación a un proyecto democratizador (BETANCUR, 2008; BORDOLI; CONDE, 2016); 3) ciertas especificidades de la tradición y arquitectura institucional del sistema político-administrativo uruguayo (especialmente su carácter gradualista, pluralista y centralista) (BETANCUR, 2012; BORDOLI; CONDE, 2016; LANZARO, 2004); 4) la oposición por parte de organizaciones sociales a la agenda neoliberal (MOREIRA, 2001); y 5) la limitada influencia del BID y del Banco Mundial en materia de asesoramiento técnico.

No obstante, se detecta en los últimos años un cambio significativo en el orden discursivo. Desde diferentes fuentes se asocia la provisión educativa privada con mayores niveles de eficiencia (a la vez que se disocia educación pública de educación de calidad), se cuestiona el vínculo entre educación pública y movilidad social, y se expande la percepción de una crisis educativa vinculada a las especificidades de la arquitectura institucional uruguaya (BETANCUR, 2008; BORDOLI; CONDE, 2016).

Si bien este cambio discursivo no se ha traducido en un crecimiento inmediato del sector privado, contribuye a la creación de un clima propicio a las propuestas de reforma educativa de corte privatizador, por medio de un giro en las prioridades de la agenda política y la emergencia de un nuevo sentido común sobre la educación pública entre la clase política. Así, y desde la década de 2000, crecen las voces a favor de los esquemas de gestión privada y financiamiento público basados en la libertad de elección escolar (BETANCUR, 2012; D'AVENIA, 2013). Aunque tales propuestas se asocian de forma más clara al Partido Colorado y a colectivos ligados a la Iglesia Católica, esta agenda ha acabado permeando en casi todo el espectro político -siendo progresivamente asumida por facciones de la actual coalición de gobierno (el Frente Amplio), situado en el centro izquierda (BORDOLI; CONDE, 2016).

Finalmente, cabe tener en cuenta que, más allá del bloqueo actual de las políticas promercado en el ámbito educativo, otras leyes de carácter extra-educativo han contribuido a crear un entorno propicio a la iniciativa privada en materia de provisión de servicios sociales. Específicamente, la reforma tributaria de 2007 estableció una exoneración fiscal del 82,5\% para donaciones empresariales a entidades educativas y sociales. Esta ley no se ha traducido en un aumento claro de las iniciativas educativas privadas, pero ha tenido un efecto de consolidación de dinámicas preexistentes. A la vez, ha alentado el establecimiento, de carácter experimental, de un reducido núcleo de nuevos establecimientos de gestión privada y de acceso gratuito, típicamente dirigidas a alumnado en situación de vulnerabilidad socioeconómica (BORDOLI; CONDE, 2016). Así mismo, la nueva ley presupuestaria de 2015 establece un marco notablemente 
favorable a la cooperación público-privada en materia de infraestructura, susceptible de experimentar un escalamiento en materia de gestión de centros educativos.

\section{Conclusiones y prospectiva del estudio}

La privatización de la educación es un fenómeno global que se presenta de forma especialmente acusada en Latinoamérica. Ahora bien, el avance del sector privado en la región responde a una realidad en absoluto monolítica. El desarrollo de un amplio abanico de trayectorias de privatización educativa en la región (véase la Tabla 1) se comprende sólo a la luz de una serie de especificidades y contingencias (políticas, económicas e institucionales) que cuentan con un carácter marcadamente endógeno.

La literatura existente sobre privatización educativa en América Latina no se distribuye de forma equitativa en términos territoriales. Algunos casos, como Argentina, Colombia y, en especial, Chile, se encuentran ampliamente documentados desde una gran variedad de enfoques. Por el contrario, casos de gran interés desde la perspectiva de la privatización educativa se encuentran particularmente infra documentados. Bolivia, Ecuador, Panamá, Paraguay, Uruguay, y Venezuela son algunos ejemplos de ello. En estos casos, la literatura disponible suele ser muy limitada, proviene de fuentes no académicas o es directamente inexistente. En cualquier caso, la escasez relativa de literatura para ciertas áreas geográficas no refleja tanto la poca relevancia de la temática de la privatización educativa como la pervivencia de zonas centrales y periféricas en lo que respeta a la producción de conocimiento y diseminación científica.

A pesar de que hemos elaborado las trayectorias hacia la privatización educativa a partir de casos nacionales, cabe tener en cuenta que, como se señalaba en el apartado anterior, la privatización educativa implica normalmente la convergencia de elementos, distintivos de más de una trayectoria. Un ejemplo ilustrativo de este carácter mixto es el caso de la República Dominicana, donde la expansión del sector privado se explica por la combinación de un sistema de alianzas público-privadas de matriz histórica y el posterior desarrollo de la modalidad de escuelas privadas de bajo costo en áreas urbanas. En este sentido, la referencia a ciertos países como casos paradigmáticos no excluye que gran parte de las dinámicas descritas puedan ser observadas también en otros contextos. Además, en la mayoría de los sistemas educativos de la región se produce una cierta sedimentación y solapamiento de diferentes políticas y procesos proprivatización. La falta de capacidad institucional por parte de ciertos Estados para imponer y sostener reformas en el tiempo da a los sistemas educativos latinoamericanos una fisonomía fragmentada, construida históricamente, y que refleja la convivencia de distintas y, por momentos contradictorias, reformas educativas.

Finalmente, cabe destacar que, contra la tendencia general en la región, en algunos casos la matrícula privada relativa ha experimentado una tendencia a la baja durante las últimas décadas. Este es el caso en particular de Bolivia, donde el retroceso moderado de la escolarización privada dibuja una posible trayectoria hacia una desprivatización más profunda. En este país, la desprivatización educativa sería el resultado de un refuerzo del sistema público a nivel de formación docente y condiciones escolares, un mayor control público sobre el sector privado (que limita buena parte de los incentivos económicos a la oferta de educación privada), y políticas de transferencia de renta condicionadas a 
la escolarización en el sistema público (MARCO NAVARRO, 2012; MOGROVEJO, 2010; SCHIPPER, 2014). No obstante, sería necesario generar más evidencias sobre las causas de la contención del sector privado en este país así como, en términos más generales, la realización de nuevos estudios que indaguen sobre cómo y bajo qué circunstancias se pueden impulsar procesos de desprivatización educativa desde la política pública.

\section{Referencias}

ALMEIDA, Ana Maria et al. A educação privada na Argentina e no Brasil. Educação e Pesquisa, São Paulo, v. 43, n. 4, p. 939-956, 2017.

BALARIN, María. La privatización por defecto y el surgimiento de las escuelas privadas de bajo costo en el Perú ¿Cuáles son sus consecuencias? RASE: Revista de la Asociación de Sociología de la Educación, Madrid, v. 9, n. 2, p. 181-196, 2016.

BALL, Stephen J. Privatising education, privatising education policy, privatising educational research: Network governance and the 'competition state'. Journal of Education Policy, London, v. 24, n. 1, p. 83-9, 2009.

BALL, Stephen J.; YOUDELL, Deborah. Hidden privatisation in public education. Bruselas: Education International, 2008. Disponible en: <https://www.ei-ie.org/media_gallery/2009-00034-01-E.pdf>. Acceso en: 11 nov. 2017.

BELLEI, Cristián. Dificultades y resistencias de una reforma para des-mercantilizar la educación. RASE: Revista de la Asociación de Sociología de la Educación, Madrid, v. 9, n. 2, p. 232-247, 2016.

BELLEI, Cristián. The private-public school controversy: the case of Chile. In: CHAKRABARTI, Rajashri; PETERSON, Paul E. (Org.). School choice international: exploring public-private partnerships. Cambridge: MIT Press, 2007. p. 165-192.

BELLEI, Cristián; ORELLANA, Víctor. What does ‘education privatisation' nean? Conceptual discussion and empirical review of Latin American cases. New York: Open Society Foundations, 2014. (ESP working paper series, n. 62). Disponible en: <http://www.periglobal.org/sites/periglobal.org/files/WP62\%20What\%20 does\%20\%27Education\%20Privatisation\%27\%20mean.pdf>. Acceso en: 11 nov. 2017.

BELLEI, Cristián; VANNI, Xavier. The evolution of educational policy, 1980-2014. In: SCHWARTZMAN, Simon (Org.). Education in South America. London: Bloomsbury Academic, 2015. p. 179-200.

BETANCUR, Nicholás. Aporte para una topografía de las políticas educativas en Uruguay: instituciones, ideas y actores. Revista Uruguaya de Ciencia Política, Montevideo, v. 21, n. 1, p. 65-92, 2012.

BETANCUR, Nicholás. Las reformas educativas de los años noventa en Argentina, Chile y Uruguay: racionalidad política, impactos y legados para la agenda actual. Montevideo: Banda Oriental, 2008.

BORDOLI, Eloisa; CONDE, Stefanía. El progresivo encanto por la gestión privada: análisis de modelos de gestión público-privado en enseñanza media en Uruguay (2002-2013). Educação \& Sociedade, Campinas, v. 37, n. 134, p. 73-89, 2016. 
BOTTINELLI, Leandro. El debate sobre el crecimiento reciente de la educación privada. Buenos Aires: Ministerio de Educación de la Nación, 2013. (La educación en debate, n. 11.). Disponible en: <http:// repositorio.educacion.gov.ar/dspace/bitstream/handle/123456789/109841/educa11. pdf?sequence=1 > . Acceso en: 11 nov. 2017.

BR00KE, Nigel. 0 futuro das políticas de responsabilização educacional no Brasil. Cadernos de Pesquisa, São Paulo, v. 36, n. 128, p. 377-401, 2006.

BURCH, Patricia. Hidden markets: the new education privatization. New York: Routledge, 2009.

CABALIN, Cristian. Neoliberal education and student movements in Chile: inequalities and malaise. Policy Futures in Education, Auckland, v. 10, n. 2, p. 219-228, 2012.

CARNOY, Martin. Las políticas educacionales de Chile desde una perspectiva internacional. In: COX, Cristián (Org.). Políticas educacionales en el cambio de siglo: la reforma del sistema escolar de Chile. Santiago de Chile: Universitaria, 2003. p. 115-124.

CASTILLO, Melba; MARTíNEZ, Julio. Programa de escuelas autogestionadas en Nicaragua: lecciones de una experiencia. Buenos Aires: IIPE-UNESCO, 2015. Disponible en: <http://www.buenosaires.iipe.unesco.org/ sites/default/files/Programa\%20Escuelas\%20Aut\%C3\%B3nomas.docx>. Acceso en: 11 nov. 2017.

CLADE - Campaña Latinoamericana por el Derecho a la Educación. Mapeo sobre tendencias de la privatización de la educación en América Latina y el Caribe. São Paulo: CLADE, 2014. Disponible en:<https://v2.campanaderechoeducacion.org/es/publicaciones/clade/publicaciones-tematicas. html?download=316\%3Amapeo-sobre-tendencias-de-la-privatizacion-de-la-educacion-en-americalatina-y-el-caribe>. Acceso: 11 nov. 2017.

COUPLAND, Christine; CURRIE, Graeme. BOYETT, Inger. New public management and a modernization agenda: implications for school leadership. International Journal of Public Administration, New York, v. 31 , n. 9, p. 1079-1094, 2008.

COX, Cristián; ÁVALOS, Beatrice. Educational change programmes and international co-operation: the case of Chile. In: KING, Kenneth; BUCHERT, Lene (Org.). Changing international aid to education: global patterns and national contexts. París: Unesco, 1999. p. 280-297.

CUCUZZA, Héctor. Estudios de historia de la educación durante el primer peronismo, 1943-1955. Buenos Aires: Libros del Riel, 1997.

CUÉLLAR-MARCHELLI, Helga. Decentralization and privatization of education in El Salvador: assessing the experience. International Journal of Educational Development, London, v. 23, n. 2, p. 45-166, 2003.

DA COSTA, Marcio; KOSLINSKI, Marianne Campelo; COSTA, Lygia. Educational inequality and social stratification in Brazil. In: PEILIN, Li et al. (Ed.). Handbook on social stratification in the BRIC countries: change and perspective. London: World Scientific, 2013. p. 501-523.

DA COSTA, Néstor. La laicidad uruguaya. Archives de Sciences Sociales des Religions, París, n. 146, p. 137-155, 2009. 
DA SILVA, Luís Gustavo Alexandre; ALVES, Miriam Fábia. Gerencialismo na escola pública: contradições e desafios concernentes à gestão, à autonomia e à organização do trabalho escolar. Revista Brasileira de Política e Administração da Educação, Goiânia, v. 28, n. 3, p. 665-681, 2012.

D’AVENIA, Lucas. Evolución de la estructura institucional y de la gestión de la educación pública en los últimos 50 años: desafíos para la próxima década. Informe final. Montevideo: INEE-CIDE, 2013. Disponible en: <https://www.ineed.edu.uy/images/pdf/evolucion-estructura-inst.pdf>. Acceso en: 11 nov. 2017.

DAY ASHLEY, Laura et al. The role and impact of private schools in developing countries: education rigorous literature review. London: DFID, 2014. Disponible en: <https://eppi.ioe.ac.uk/cms/Portals/0/ PDF\%20reviews\%20and\%20summaries/Private\%20schools\%202014\%20Day\%20Ashley\%20report. pdf?ver=2015-06-25-141203-947>. Acceso en: 11 nov. 2017.

DELANNOY, Francoise. Education reforms in Chile, 1980-1998: a lesson in pragmatism. Washington, DC: World Bank, 2000. (Country studies: education reform and management publication series; v. 1, n. 1.). Disponible en: <http://www-wds.worldbank.org/external/default/WDSContentServer/WDSP/IB/2000/09/0 1/000094946_00081805551098/Rendered/PDF/multi_page.pdf>. Acceso en: 11 nov. 2017.

DE LUCA, Romina. Brutos y baratos: descentralización y privatización en la educación argentina: 19552001. Buenos Aires: RYR, 2008.

DEMOMBYNES, Gabriel; HOLLAND, Peter; GIANMARCO, Leon. Students and the market for schools in Haiti. Washington, DC: World Bank, 2010. (World Bank policy research working paper; n. 5503). Disponible en: <https://www.openknowledge.worldbank.org/bitstream/handle/10986/3985/WPS5503. txt?sequence=2>. Acceso en: 11 nov. 2017.

DINIECE. Anuarios estadísticos. Buenos Aires: DiNIECE, 2016. Disponible en: http://portales.educacion.gov. ar/diniece/2014/05/24/anuarios-estadisticos/. Acceso en: 11 nov. 2017.

EDWARDS, Brent. Rising from the ashes: how the global education policy of community-based management was born from El Salvador's civil war. Globalisation, Societies and Education, Abingdon, v. 13, n. 3, p. 411-432, 2015.

FLORES, Ramón. La escuela básica en la zona marginal de Santo Domingo: un estudio comparativo de la organización industrial del servicio de educación en la República Dominicana. Washington, DC: InterAmerican Development Bank, 1997. Disponible en: <https://publications.iadb.org/handle/11319/6099>. Acceso en: 11 nov. 2017.

FOURCADE-GOURINCHAS, Marion; BABB, Sarah L. The rebirth of the liberal creed: paths to neoliberalism in four countries. American Journal of Sociology, Chicago, v. 108, n. 3, p. 533-579, 2002.

FRIEDMAN, Milton. The role of government in education. In: SOLO, Robert A. (Org.). Economics and the public interest. New Jersey: Rutgers University Press, 1955. p. 123-144.

GAMALLO, Gustavo. La 'publificación' de las escuelas privadas en Argentina. Revista Sociedad Argentina de Análisis Político, Buenos Aires, v. 9, n. 1, p. 43-74, 2015. 
GAMALLO, Gustavo. Mercantilización del bienestar. hogares pobres y escuelas privadas. Revista de Instituciones, Ideas y Mercados, Buenos Aires, n. 55, p. 189-233, 2011.

GANIMIAN, Alejandro J. Why do some school-based management reforms survive while others are reversed? The cases of Honduras and Guatemala. International Journal of Educational Development, London, v. 47, p. 33-46, 2016.

GAURI, Varun. School choice in Chile: two decades of educational reform. Pittsburgh: University of Pittsburgh Press, 1998.

GERTLER, Paul J.; PATRINOS, Harry Anthony; RUBIO-CODINA, Marta. Empowering parents to improve education: evidence from rural Mexico. Journal of Development Economics, Amsterdam, v. 99, n. 1, p. 68-79, 2012.

GOTTAU, Verónica; MOSCHETTI, Mauro. El sistema educativo argentino entre la privatización interna y la privatización abierta: trayectorias divergentes y desigualdades. Cadernos de Pesquisa: Pensamento Educacional, Tuiuti, v. 10, n. 26, p. 182-202, 2015.

GOUGH, David; THOMAS, James; OLIVER, Sandy. Clarifying differences between review designs and method. Systematic Reviews, London, v. 1, n. 28, 2012. Disponible en: <http://link.springer.com/content/ pdf/10.1186\%2F2046-4053-1-28.pdf>. Acceso en: 11 nov. 2017.

GUZMÁN, José; MEZA, Darlyn; DE VARELA, Lorena. Educo: a community-managed education program in rural El Salvador (1991-2003). Washington, DC: World Bank, 2004. Disponible en: <https:// openknowledge.worldbank.org/bitstream/handle/10986/10355/307310ENGLISHOen0breve0Educo. pdf? sequence=1\&isAllowed=y>. Acceso en: 11 nov. 2017.

GUZMÁN, Rolando; CRUZ, Consuelo. Niños, niñas y adolescentes fuera del sistema educativo en la República Dominicana. Santiago de Chile: Foro Socioeducativo, 2009. Disponible en: <http://www.gcpareto.com/ NNA.pdf>. Acceso en: 11 nov. 2017.

HÄRMÄ, Joanna. Access or quality? Why do families living in slums choose low-cost private schools in Lagos, Nigeria? Oxford Review of Education, Oxford, v. 39, n. 4, p. 548-566, 2013.

INEED. Instituto Nacional de Evaluación Educativa. OECD review of policies to improve the effectiveness of resource use in schools (School Resources Review). Country Background Report for Uruguay, Montevideo, 2015. Disponible en: <https://www.oecd.org/edu/CBR_URUGUAY_ENGLISH_FINAL.pdf>.Acceso en: 11 nov. 2017.

JESSOP, Bob. The future of the capitalist state. London: Polity, 2002.

JUDZIK, Darío; MOSCHETTI, Mauro. ¿Una segunda fase de privatización de la matrícula escolar? Los sectores populares y la educación privada en la ciudad de Buenos Aires. RASE: Revista de la Asociación de Sociología de la Educación, Madrid, v. 9, n. 2, p. 197-211, 2016.

LANZARO, Jorge. La reforma educativa en Uruguay (1995-2000): virtudes y problemas de una iniciativa heterodoxa. Santiago de Chile: CEPAL, 2004. Disponible en: <http://repositorio.cepal.org/bitstream/ handle/11362/6081/1/S047549_es.pdf>. Acceso en: 11 nov. 2017. 
MANCEBO, María Ester. Schooling in Uruguay. In: GVIRTZ, Silvina; BEECH, Jason (Org.). Going to school in Latin America. Westport: Connecticut; London: Greenwood Press, 2008. p. 303-321.

MARCO NAVARRO, Flavia. El Bono Juancito Pinto del Estado plurinacional de Bolivia: programas de transferencias monetarias e infancia. Santiago de Chile: CEPAL, 2012. Disponible en: <http://repositorio. cepal.org/hitstream/handle/11362/4005/S1200591_es.pdf?sequence=1\&isAllowed=y >. Acceso en: 11 nov. 2017.

MAROY, Christian. Regulation and inequalities in European education systems: rapport de recherche. Louvain-La-Neuve: GIRSEF, 2004. Disponible en: <https://cdn.uclouvain.be/public/Exports\%20reddot/ girsef/documents/reguleducnetwork_VF_10dec041.pdf>. Acceso en: 11 nov. 2017.

MCNULTY, Brendan. The education of poverty: rebuilding Haiti's school system after its 'total collapse'. The Fletcher Forum of World Affairs, Medford-Massachusetts, v. 35, n. 1, p. 109-126, 2011. Disponible en: <http://fletcher.tufts.edu/Fletcher-Forum/Archives/ /media/Fletcher/Microsites/Fletcher\%20Forum/ PDFs/2011 winter/McNulty.pdf>. Acceso en: 11 nov. 2017.

MEZZADRA, Florencia; RIVAS, Axel. Aportes estatales a la educación de gestión privada en la provincia de Buenos Aires. Buenos Aires: CIPPEC, 2010. (Documentos de trabajo CIPPEC). Disponible en: <https://www. cippec.org/wp-content/uploads/2017/03/2525.pdf>. Acceso en: 11 nov. 2017.

MIZALA, Alejandra. La economía política de la reforma educacional en Chile. Santiago de Chile: Corporación de Estudios para Latinoamérica, 2007. (Estudios socio/económicos; n. 36). Disponible en: <http://cieplan. cl/media/publicaciones/archivos/153/Capitulo_1.pdf>. Acceso en: 11 nov. 2017.

MOGROVEJO, Rodrigo. Las políticas educativas en Bolivia como estrategias de lucha contra la pobreza: diversidad de enfoques e influencia de la cooperación internacional (1994-2010). San Sebastián: UPV/EHU, 2010. 582 p. Tesis (Doctorado) - Programa de Doctorado en Estudios sobre Desarrollo. Instituto de Estudios sobre el Desarrollo y Cooperación Internacional (Hegoa), Universidad del País Vasco, San Sebastián, 2010.

MORDUCHOWICZ, Alejandro. Private education: funding and (de) regulation in Argentina. Washington, DC: National Center for the Study of Privatization in Education, 2001. Disponible en: <http://files.eric.ed.gov/ fulltext/ED465215.pdf>. Acceso en: 11 nov. 2017.

MORDUCHOWICZ, Alejandro; IGLESIAS, Gustavo. Auge y avance de los subsidios estatales a las escuelas privadas en la Argentina. In: PERAZZA, Roxanna (Org.). Mapas y recorridos de la educación de gestión privada en la Argentina. Buenos Aires: Aique, 2011. p. 34-56.

MOREIRA, Constanza. La reforma de Estado en Uruguay: cuestionando el gradualismo y la heterodoxia. In: CALAME, Pierre; TALMANT, André (Org.). Con el Estado en el corazón: el andamiaje de la gobernancia. Montevideo: Trilce, 2001. p. 167-202.

MOSCHETTI, Mauro. Private education supply in disadvantaged areas of the city of Buenos Aires and 'low-fee private cchooling': comparisons, contexts, and implications. EPAA-AAPE, Arizona, v. 23, n. 126, p. 1-26, 2015. 
NAHON, Cecilia; Política social y acumulación de capital en la Argentina: la fragmentación regresiva de la educación, la salud y la previsión social en la década del noventa. FACES: Revista de la Facultad de Ciencias Económicas y Sociales, Universidad Nacional de Mar del Plata, Mar del Plata, v. 8, n. 15, p. 23-70, 2002.

NARODOWSKI, Mariano. School choice and quasi-state monopoly in education systems in Latin America: the case of Argentina. In: FORSEY, Martin; DAVIES, Scott; WALFORD, Geoffrey (Org.). The globalisation of school choice? Oxford: Symposium Books, 2008. p. 131-144.

NARODOWSKI, Mariano; ANDRADA, Myrian. The privatization of education in Argentina. Journal of Education Policy, London, v. 16, n. 6, p. 585-595, 2001.

NARODOWSKI, Mariano; MOSCHETTI, Mauro. The growth of private education in Argentina: evidence and explanations. Compare: A Journal of Comparative and International Education, London, v. 45, n. 1, p. 47-69, 2015.

O'KEEFE, Joy. The role of an epistemic community in Haitian education reform postearthquake 2010. 2013. 57 p. Tesina (Maestría) - Program in Cultural and Educational Policy Studies, School of Education, Loyola University, Chicago, 2013.

PAREDES, Ricardo. UGARTE, Gabriel. Should students be allowed to miss? Munich: Munich Personal RePEc Archive, 2009. (MPRA paper; n. 15583.). Disponible en: <https://mpra.ub.uni-muenchen.de/15583/1/ MPRA_paper_15583.pdf>. Acceso en: 11 nov. 2017.

PEREYRA, Ana. La fragmentación de la oferta educativa en América Latina: la educación pública vs. la educación privada. Perfiles Educativos, México, v. 30, n. 120, p. 132-146, 2008.

PEROSA, Graziela Serroni; DANTAS, Adriana Santiago Rosa. A escolha da escola privada em famílias dos grupos populares. Educação e Pesquisa, São Paulo, v. 43, n. 4, p. 987-1004, 2017.

PHILLIPSON, Bob. Low-cost private education: impacts on achieving universal primary education. London: Commonwealth Secretariat, 2008.

PUIGGRÓS, Adriana. Qué pasó en la educación argentina: breve historia desde la conquista hasta el presente. Buenos Aires: Galerna, 2003.

REIS DE FIGUEIREDO, Gil Vicente. Desafios da atual conjuntura no Brasil. In: SEMINARIO SOBRE PROVATIZACIÓN Y COMERCIALIZACIÓN DE LA EDUCACIÓN EM AMÉRICA LATINA, 2016, San José de Costa Rica. Anales... San José de Costa Rica: [s. n.], 2016.

REZENDE PINTO, José Marcelino. Uma análise da destinação dos recursos públicos, direta ou indiretamente, ao setor privado de ensino no Brasil. Educação \& Sociedade, Campinas, v. 37, n. 134, p. 133-151, 2016.

SANTIZO RODALL, Claudia; MARTIN, Christopher James. School-based management and citizen participation: lessons for public education from local educational projects. Journal of Education Policy, London, v. 24, n. 3, p. 317-333, 2009.

SARANGAPANI, Padma M.; WINCH, Christopher. Tooley, Dixon and Gomathi on private education in Hyderabad: a reply. Oxford Review of Education, Oxford, v. 36, n. 4, p. 499-515, 2010. 
SCHIPPER, Catharina Jenny. Teacher training in Bolivia and the implementaion of the 2010 education reform. 2014. 97 p. Tesina (Maestría) - International Development Studies, Graduate School of Social Sciences, University of Amsterdam, Amsterdam, 2014.

SIQUEIRA, Ana Rita; NOGUEIRA, Maria Alice de Lima Gomes. Focalizando um segmento específico da rede privada de ensino: escolas particulares de baixo custo. Educação e Pesquisa, São Paulo, v. 43, n. 4, p. 1005-1022, 2017.

SRIVASTAVA, Prachi. The shadow institutional framework: towards a new institutional understanding of an emerging private school sector in India. Research Papers in Education, London, v. 23, n. 4, p. 451-475, 2008.

TEJADA, Argelia. ¿Educación privada y gratuita? Acento, Santo Domingo, 2015. Disponible en: $<$ http://acento.com.do/2015/opinion/8308647-educacion-privada-y-gratuita>. Acceso en: 11 nov. 2017.

TERMES, Andreu et al. Public-private partnerships in Colombian education: the equity and quality implications of "Colegios en concesión". New York: Open Society Fundations, 2015. (ESP working paper; 66). Disponible en: <http://www. periglobal. org/role-state/document/public-private-partnerships-colombian-educationequity-and-qualityimplications->. Acceso em: 14 jul. 2016.

TOOLEY, James; DIXON, Pauline. 'De facto' privatisation of education and the poor: implications of a study from sub-Saharan Africa and India. Compare, London, v. 36, n. 4, p. 443-462, 2006.

UNESCO-UIS. Indicadores - Educación. 2016. Disponible en: <http://uis.unesco.org/>. Acceso en: 11 nov. 2017.

VALENZUELA, Juan Pablo; BELLEl, Cristian; RÍOS, Danae de los. Socioeconomic school segregation in a market-oriented educational system: the case of Chile. Journal of Education Policy, London, v. 29, n. 2, p. 217-241, 2014.

VALLAS, Paul; PANKOVITS, Tressa. Education in the wake of natural disaster. Port-au-Prince: Wilson Center, 2010. Disponible en: <https://www.wilsoncenter.org/sites/default/files/PAGE_130918_Vallas\%20 Report\%20ll\%20Haiti_1104.pdf>. Acceso en: 11 nov. 2017.

VERGER, Antoni; FONTDEVILA, Clara; ZANCAJO, Adrián. The privatisation of education: a political economy of global education reform. New York: Teachers College Press, 2016.

VIOR, Susana E.; RODRÍGUEZ, Laura R. La privatización de la educación argentina: un largo proceso de expansión y naturalización. Pro-Posições, Campinas, v. 23, n. 2, p. 91-104, 2012.

WALFORD, Geoffrey. Low-fee private schools in England and in less economically developed countries: what can be learnt from a comparison? Compare, London, v. 41, n. 3, p. 401-413, 2011.

WORLD BANK DATA. Estadísticas - Educación. 2016. Disponible en: <http://data.worldbank.org/>. Acceso en: 11 nov. 2017.

YITZACK PAVÓN, Fernando. Improving educational quality in Honduras: building a demand-driven education market. Journal of Public and International Affairs, Princeton, v. 19, n. 1, p. 194-213, 2008. 
Recibido en: 15.11 .2017

Revisiones en: 20.02 .2018 Aprobado en: 24.04.2018

Mauro C. Moschetti es profesor asociado e investigador predoctoral del Departamento del Pedagogía Sistemática y Social de la Universitat Autònoma de Barcelona y miembro del centro de investigación Globalization, Education and Social Policies (GEPS).

Clara Fontdevila es investigadora predoctoral en el Departamento de Sociología de la Universitat Autònoma de Barcelona. Ha colaborado en proyectos de investigación con Education International, Fundació Jaume Bofill y Open Societies Foundations. Sus áreas de interés son los mercados educativos y la gobernanza global de la educación.

Antoni Verger es profesor del Departamento de Sociología de la Universitat Autònoma de Barcelona, miembro fundador del centro de investigación Globalization, Education and Social Policies (GEPS) y subdirector del programa Erasmus + Máster Education Policies for Global Development (GLOBED). 